Ann. Biol. anim. Bioch. Biophys., 1968, 8 (2), 3I3-3I5.

\title{
ÉLÉVATION DU TAUX DE LH DANS LE PLASMA SANGUIN DU BELLIER APRÈS CASTRATION
}

\author{
J. PELLETIER \\ avec la collaboration technique de G. Fouilleul et Christiane Rouel \\ Laboratoire de Physiologie de la Reproduction, \\ Centre de Recherches vétérinaires et zootechniques, 37 - Nouzilly, \\ Institut national de la Recherche agronomique
}

Le fonctionnement du système hypothalamo-hypophysaire chez le Bélier après castration doit être précisé : en effet, à la différence du Rat, nous n'avons pu mettre en évidence chez le bélier adulte castré depuis 3 mois, une augmentation ni de l'activité LRF dans l'hypothalamus ni de la teneur hypophysaire en LH : l'activité LRF, exprimée en $\mu \mathrm{g}$ de LH-NIH-S8 libérée par mg d'hypophyse incubée in vitro, a été trouvée de $0,74 \mathrm{I}$ chez les béliers entiers et de $0,45^{\circ} \mathrm{chez}$ les béliers castrés ; les teneurs hypophysaires en LH déterminées par le dosage de ParLow (1958), et exprimées en $\mu \mathrm{g}$ de LH-NIH-S8 par mg d'hypophyse sèche étaient respectivement de 20,4 et 21,5 ; les poids hypophysaires étaient semblables dans les deux séries expérimentales. Enfin les cellules hypophysaires, dites " en chaton de bague ", caractéristiques de la castration chez le Rat sont absentes (M. DuBors, communication personnelle).

Dans le plasma, McDonald et ClegG (1966) ne trouvent pas d'augmentation de la LH après castration du Bélier, en utilisant la technique de Parlow (1958). Cependant nous avons montré que pour le dosage de la LH plasmatique, la technique de Parlow pouvait être critiquable : en effet l'injection intraveineuse d'un plasma d'ovin aux rattes réceptrices peut provoquer un stress chez ces animaux et induire une diminution non spécifique de l'acide ascorbique ovarien (Pelletier, i9641965).

Aussi avons-nous repris ce problème en dosant la LH dans le plasma à l'aide de la technique radio; immunologique récemment décrite (Pelletier, Kann, Dolais et Rosselin, 1968).

Des prélèvements de sang ont été effectués, par la jugulaire entre io et I I heures du matin, chez 6 béliers $I l e$-de-France, âgés de 15 mois, durant les 7 premiers jours après la castration (expérience $\mathrm{A}$ ), puis durant 18 semaines après cette opération chez des béliers plus âgés (4-5 ans, expérience B) ; 6 autres béliers entiers d'âge comparable aux béliers expérimentaux ont servi de témoins. 
Les résultats exprimés en $\mu \mathrm{g}$ de $\mathrm{LH} \cdot \mathrm{M}_{1}\left({ }^{1}\right) / \mathrm{ml}$ de plasma sont les suivants :

\begin{tabular}{|c|c|c|c|}
\hline & \multirow{2}{*}{$\begin{array}{l}\text { Temps après } \\
\text { castration }\end{array}$} & \multicolumn{2}{|c|}{ LH plasmatique chez le Bélier } \\
\hline & & normal & castré \\
\hline Expérience $\mathbf{A}$ & $\begin{array}{l}0 \\
6 \text { heures } \\
12- \\
24- \\
2 \text { jours } \\
3- \\
4- \\
5- \\
6- \\
7-\end{array}$ & $\begin{array}{r}3,3 \pm 0,5 \\
4,1 \pm 0,6 \\
3,5 \pm 0,4 \\
3,4 \pm 0,6 \\
3,7 \pm 0,6 \\
3,7 \pm 0,8 \\
5,2 \pm 1,7 \\
3,3 \pm 0,9 \\
4,9 \pm 1,7\end{array}$ & $\begin{array}{r}3,3 \pm 0,5 \\
3,7 \pm 0,4 \\
7,2 \pm 1,5 \\
6,7 \pm 0,6 \\
8,1 \pm 0,9 \\
6,9 \pm 0,8 \\
7,9 \pm 0,9 \\
13,0 \pm 1,7 \\
9,1 \pm 1,2 \\
14,8 \pm 3,3\end{array}$ \\
\hline Expérience $\mathbf{B}$ & \begin{aligned} & \multicolumn{1}{l}{$\mathrm{T}_{0}$} \\
& 1 semaine \\
& 2 semaines \\
& 4- \\
& 6- \\
& 10- \\
& 14- \\
& 18-\end{aligned} & $\begin{array}{l}7,2 \pm 0,7 \\
4,2 \pm 1,4 \\
6,3 \pm 0,5 \\
5,7 \pm 0,5 \\
8,6 \pm 1,0 \\
8,8 \pm 1,7 \\
8,2 \pm 0,7 \\
6,7 \pm 1,3\end{array}$ & $\begin{array}{r}7,2 \pm 0,7 \\
19,0 \pm 3,6 \\
16,2 \pm 1,5 \\
17,6 \pm 2,3 \\
22,3 \pm 2,8 \\
21,7 \pm 3,7 \\
23,6 \pm 3,4 \\
28,1 \pm 4,7\end{array}$ \\
\hline
\end{tabular}

Les résultats obtenus sont nets : la LH augmente dans le plasma de l'animal castré d'environ $3^{00} \mathrm{p}$. 100. Cette augmentation décelable dès 12 heures après la castration $(\mathbf{P}<0,05)$, est brutale durant la première semaine, puis devient plus faible durant les semaines suivantes.

On peut conclure de ces résultats qu'un mécanisme analogue à celui décrit chez le Rat existe bien chez le Bélier : en l'absence d'androgènes, le système hypothalamo-hypophysaire possède une activité plus élevée se traduisant par une décharge importante de l'hormone LH dans le sang. Toutefois ces phénomènes ne s'accompagnent pas comme chez le Rat d'une augmentation des teneurs du LRF de l'hypothalamus ni de la LH de l'hypophyse.

Reçu pour publication en avril 1968.

\section{SUMMARY}

\section{INCREASE IN THE LH PLASMA LEVEL OF THE RAM AFTER CASTRATION}

LH plasma level was estimated by radio-immunological method in $\mathbf{2} 2$ Ile-de-France rams following castration.

LH increased very rapidly during the first week, and more slowly in the subsequent weeks. Plasma LH attained a value 3 times higher than that of the controls.

The first increase was detected as early as $\mathrm{I} 2$ hours after castration.

(1) La LH-M $\mathrm{M}_{1}$ a été préparée par JuTisz et CourTE (1968) que nous remercions ; elle possède une activité de 1,8 unité de $L H-N I H-S_{1}$ par milligramme. 


\section{RÉFÉRENCES BIBLIOGRAPHIQUES}

Jutrsz M., CourTe C., rg68. Gen. Comp. Endocr. (sous presse).

McDonald P. G., ClegG M. T., 1966. Some factors affecting gonadotropin levels in Sheep. Proc. Soc. Exp. Biol. Med., 121, 482-485.

ParLOW A. G., 1958. A rapid bioassay method for $\mathrm{LH}$ and factors stimulating $\mathrm{LH}$ secretion. Feder. Proc., $17,402$.

Pelletier J., r964. Dosage de l'hormone stimulant l'interstitielle (ICSH) dans le sang par la méthode de l'acide ascorbique ovarien. C. R. Acad. Sci., 258, 5979-5981.

Pelletier J., 1965. Effet du plasma de Brebis sur la décharge de IH chez la Ratte. C.R. Acad.Sci.,260, 5624-5626.

Pelletier J., Kann G. Dolais J., Rosselin G., ig68. Dosage radio-imumnologique de l'hormone lutéinisante plasmatique chez le Mouton. C.R. Acad. Sci. (sous presse). 